TRANSACTIONS OF THE

AMERICAN MATHEMATICAL SOCIETY

Volume 350, Number 6, June 1998, Pages 2431-2455

S 0002-9947(98)02048-0

\title{
ON HOMOLOGICAL PROPERTIES OF SINGULAR BRAIDS
}

\author{
VLADIMIR V. VERSHININ
}

\begin{abstract}
Homology of objects which can be considered as singular braids, or braids with crossings, is studied. Such braids were introduced in connection with Vassiliev's theory of invariants of knots and links. The corresponding algebraic objects are the braid-permutation group $B P_{n}$ of R. Fenn, R. Rimányi and C. Rourke and the Baez-Birman monoid $S B_{n}$ which embeds into the singular braid group $S G_{n}$. The following splittings are proved for the plusconstructions of the classifying spaces of the infinite braid-permutation group and the singular braid group

$$
\begin{aligned}
& \mathbb{Z} \times B B P_{\infty}^{+} \simeq \Omega^{\infty} S^{\infty} \times S^{1} \times Y, \\
& \mathbb{Z} \times B S G_{\infty}^{+} \simeq S^{1} \times \Omega^{2} S^{2} \times W,
\end{aligned}
$$
\end{abstract}

where $Y$ is an infinite loop space and $W$ is a double loop space.

\section{INTRODUCTION}

Cohomologies of classical braid groups appeared in the papers of V. I. Arnold [Arn1], [Arn2]. From the very beginning their study was connected with various mathematical disciplines and questions. We mention that originally V. I. Arnold introduced these cohomologies because he found there in dimensions of the form $2^{n}-1$ the obstructions for the problem of representability of algebraic functions of several variables by means of superpositions of algebraic functions of fewer variables. In the work of Fred Cohen [CF1], [CF2], [CF3], [CLM] cohomologies of braid groups were studied in connections with the theory of configuration spaces, loop spaces, Araki-Kudo-Dyer-Lashof operations and Thom spectra. The theorem of Graeme Segal [S1], which establishes connections between homologies of the infinite braid group and the double loop space over the two-dimensional sphere is the analogue of the theorem of M. Barratt, S. Priddy and D. Quillen [Bar], [P] on relations between homologies of the infinite symmetric group and the infinite loop space $\Omega^{\infty} S^{\infty}$. This makes a bridge to the work of Mark Mahowald [Mah1], [Mah2] on homotopy theory.

Starting from E. Artin [Art1], [Art2], the braid groups themselves were connected with problems of low-dimensional topology. This idea was illuminated by the construction of the Jones polynomial for links [J]. New invariants of knots and links, invented by V. A. Vassiliev [V], attracted a lot of attention in mathematics, as probably the most powerful invariants known up to now. In attempts to interpret these invariants through braids, according to the general philosophy of V. A. Vassiliev of extending the set of basic objects to objects with simple singularities, the notion

Received by the editors August 20, 1996.

1991 Mathematics Subject Classification. Primary 20J05, 20F36, 20F38, 18D10, 55P35.

Key words and phrases. Braid group, permutation group, homology, classifying space, loop space. 
of singular braids was introduced: the Baez-Birman monoid $S B_{n}$ [Bae], [Bi] and the braid-permutation group of R. Fenn, R. Rimányi and C. Rourke $B P_{n}$ [FRR1], [FRR2]. It seems that singular braids also have broad relations with various fields of mathematics. J. C. Baez discusses in [Bae] connections of the monoid of singular braids with Chern-Simons perturbation theory and other questions of mathematical physics. The braid-permutation group is defined as a subgroup of the group of automorphisms of the free group and has the interpretation as a group of singular braids, or welded braids as the authors call them. It can also be interpreted as the automorphism group of the free quandle, and is closely related to the automorphism group of the free rack. More precisely, the latter is the wreath product of the braid-permutation group with the group of integers. In this paper we study mainly the braid-permutation group and the Baez-Birman monoid from the point of view of their homological properties.

Let $B r_{n}$ be the classical braid group on $n$ strings and $\gamma$ an inclusion of the group $\mathbb{Z}$ into $B r_{n}$ :

$$
\gamma: \mathbb{Z} \rightarrow B r_{n}
$$

when the generator of the cyclic group is mapped to one of the generators $\sigma_{i}$, say, $\sigma_{1}: \gamma(1)=\sigma_{1}$. We define the reduced braid-permutation group $\overline{B P}_{n}$ as the common factor of $B P_{n}$ and $S G_{n}$ (details are in section 2). The index $n$ in notations for groups and homomorphisms will be omitted when the case $n=\infty$ is considered. The main results of the paper are the following Theorem 1 and the similar theorem about the splitting for the singular braid group $S G_{n}$ (Theorem 3 in section 3 ).

Theorem 1. There exist maps

$$
\psi: B \Sigma \times B B r \rightarrow B B P^{+}
$$

and

$$
\beta: \Omega B\left(\coprod_{n \geq 0} B B P_{n}\right) \rightarrow \Omega^{\infty} S^{\infty} \times S^{1}
$$

such that the map $\psi$ becomes a loop map after the group completion, while the map $\beta$ is an infine loop map and it splits by the map

$$
\mathbb{Z} \times B \Sigma^{+} \times S^{1} \stackrel{I d \times(B \gamma)^{+}}{\longrightarrow} \mathbb{Z} \times B \Sigma^{+} \times B B r^{+} \stackrel{I d \times \psi^{+}}{\longrightarrow} \mathbb{Z} \times B B P^{+} .
$$

If an infinite loop space $Y$ is a fibre of the map $\beta$, then it fits for the following splitting:

$$
\Omega B\left(\coprod_{n \geq 0} B B P_{n}\right) \simeq \Omega^{\infty} S^{\infty} \times S^{1} \times Y .
$$

The same is true for the reduced braid-permutation group with one modification: the map

$$
\bar{\psi}: B \Sigma \times B B r \rightarrow B \overline{B P}^{+}
$$

becomes a double loop map after the group completion. 


\section{Groups of Automorphisms of Free Groups}

Let $F_{n}$ be the free group of rank $n$ with the set of generators $\left\{x_{1}, \ldots, x_{n}\right\}$, and let $A u t F_{n}$ be the group of automorphisms of $F_{n}$. There are the standard inclusions of the symmetric group $\Sigma_{n}$ and the braid group $B r_{n}$ into $A u t F_{n}$. They can be described as follows. Let $\xi_{i} \in A u t F_{n}, i=1,2, \ldots, n-1$, be given by the formula which describes its action on generators:

$$
\begin{cases}x_{i} & \mapsto x_{i+1} \\ x_{i+1} & \mapsto x_{i} \\ x_{j} & \mapsto x_{j}, j \neq i, i+1 .\end{cases}
$$

Let $\sigma_{i} \in A u t F_{n}, i=1,2, \ldots, n-1$, be given by the following formula:

$$
\begin{cases}x_{i} & \mapsto x_{i+1} \\ x_{i+1} & \mapsto x_{i+1}^{-1} x_{i} x_{i+1} \\ x_{j} & \mapsto x_{j}, j \neq i, i+1 .\end{cases}
$$

If we map the standard generators of the symmetric group to $\xi_{i}$ and the standard generators of the braid group to $\sigma_{i}$, then we get the monomorphisms $r$ and $t$ of groups:

$$
\begin{aligned}
& t: \Sigma_{n} \rightarrow \operatorname{AutF}_{n}, \\
& r: B r_{n} \rightarrow A u t F_{n} .
\end{aligned}
$$

We have the canonical imbeddings

$$
A u t F_{m} \times A u t F_{n} \rightarrow A u t F_{m+n} .
$$

Let $G_{n}, n=0,1, \ldots ; G_{0}=\{e\}$, be a system of subgroups of the group of automorphisms of the free group:

$$
G_{n} \leq A u t F_{n}
$$

such that $G_{m+n}$, as a subgroup of $A u t F_{m+n}$, contains the image of $G_{m} \times G_{n}$ under the map (3) for all $m$ and $n$. In this case we have a system of maps

$$
\mu_{m, n}(G): G_{m} \times G_{n} \rightarrow G_{m+n}
$$

These pairings are strictly associative; that is, the diagram

$$
\begin{aligned}
& G_{l} \times G_{m} \times G_{n} \stackrel{I d \times \mu_{m, n}}{\longrightarrow} G_{l} \times G_{m+n} \\
& \downarrow \mu_{l, m} \times I d \quad \downarrow \mu_{l, m+n} \\
& G_{l+m} \times G_{n} \stackrel{\mu_{l+m, n}}{\longrightarrow} G_{l+m+n}
\end{aligned}
$$

is commutative. Suppose now that for every $m$ the group $G_{m}$ contains the symmetric group $\Sigma_{m}$, canonically imbedded in $A u t F_{m}$. In this case the system of groups $\left\{G_{n}\right\}$ generates a $\Gamma$-category in the sense of G. Segal [S2]. This $\Gamma$-category leads to a $\Gamma$-space and then to a spectrum [S2]. But we shall look at it more directly. Really, a strict monoidal (tensor) category $\mathcal{G}$ is defined in a usual way. Its objects $\{\overline{0}, \overline{1}, \ldots\}$ correspond to the integer numbers from 0 to infinity, and morphisms are defined by the formula

$$
\operatorname{hom}(\bar{k}, \bar{l})= \begin{cases}G_{k}, & \text { if } \quad k=l \\ \emptyset, & \text { if } \quad k \neq l\end{cases}
$$


The product in $\mathcal{G}$ is defined on objects by the sum of numbers and on morphisms by the pairing $\mu$. The category $\Sigma$ corresponding to symmetric groups has a symmetry [ML, p. 180], that is, the system of isomorphisms

$$
s_{\bar{m}, \bar{n}}: \overline{m+n} \cong \overline{n+m},
$$

natural with respect to morphisms from $\bar{m}$ to itself and from $\bar{n}$ to itself, having some property of coherence and such that

$$
s_{\bar{m}, \bar{n}} s_{\bar{n}, \bar{m}}=1 \overline{m+n} .
$$

Proposition 1. If groups $G_{m}, m=0,1, \ldots$, contain the symmetric groups $\Sigma_{m}$ for all $m$, then the symmetry in the category generated by the symmetric group induces a symmetry in the category $\mathcal{G}$, so it becomes a permutative category, and the functor induced by inclusions $\Sigma_{m} \rightarrow G_{m}$ becomes a morphism between permutative categories.

Suppose that we have a system of normal subgroups $N_{m} \triangleleft G_{m}$ for all $m$, such that $N_{m+n}$, as a subgroup of $A u t F_{m+n}$, contains $N_{m} \times N_{n}$ for all $m$ and $n$. Let $H_{m}$ be the system of factor-groups

$$
H_{m}=G_{m} / N_{m}
$$

and $\mathcal{H}$ the corresponding category.

Corollary 1. The category $\mathcal{H}$ is a permutative category, and the functor

$$
\mathcal{G} \rightarrow \mathcal{H}
$$

induced by the canonical epimorphisms $G_{m} \rightarrow H_{m}$ is a morphism between permutative categories.

Let $A u t_{\infty}$ denote the union of the groups $A u t F_{n}$ under the canonical inclusions. The pairing $\mu_{m, 1}$ defines an inclusion of the group $G_{m}$ in $G_{m+1}$. In the case of the system of groups $H_{m}$ this gives a homomorphism $H_{m} \rightarrow H_{m+1}$. Then the groups $G_{\infty}$ and $H_{\infty}$ are defined as the direct limits of the systems of groups $\left\{G_{m}\right\}$ and $\left\{H_{m}\right\}$ correspondingly. Let $\coprod_{n \geq 0} B G_{n}$ and $\coprod_{n \geq 0} B H_{n}$ be the monoids of disjoint sums of the classifying spaces of $G_{n}$ and $H_{n}$, which can be identified with the classifying spaces of the corresponding categories.

Proposition 2. The classifying spaces of the categories $\mathcal{G}$ and $\mathcal{H}$ are $E_{\infty}$-spaces for which there are evident $E_{\infty}$-maps:

$$
B \Sigma \rightarrow B \mathcal{G} \rightarrow B \mathcal{H}
$$

There is the following commutative diagram of group completions:

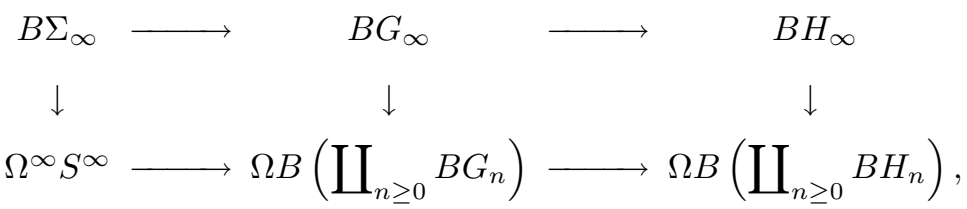

where the upper line is induced by the canonical group homomorphisms

$$
\Sigma_{\infty} \rightarrow G_{\infty} \rightarrow H_{\infty}
$$

and the bottom line consists of infinite loop maps between infinite loop spaces.

Proof. We use the theorem of Peter May and the group completion theorem (see, for example, [May] or [Ad, pp. 69, 90-91]). 
For a space $X$ of homotopy type of $C W$-complex we use the symbol $X^{+}$in the same sense as D. B. Fuks in [Fuks] uses the notion of "Quillenization". It means that $X^{+}$is a homotopy simple $C W$-complex and there exists a map

$$
q: X \rightarrow X^{+}
$$

which induces an isomorphism in homology. By the theorem of J. H. C. Whitehead $X^{+}$, if it exists, is unique up to homotopy type. So, having this approach in mind, D. Quillen's original plus construction for spaces with perfect commutator subgroup of the fundamental group is a constructive way to build $X^{+}$in this case.

We denote by $\Omega_{0} B\left(\coprod_{n \geq 0} B G_{n}\right)$ and $\Omega_{0} B\left(\coprod_{n \geq 0} B H_{n}\right)$ the connected components of the constant maps of the spaces $\Omega B\left(\coprod_{n \geq 0} B G_{n}\right)$ and $\Omega B\left(\coprod_{n \geq 0} B H_{n}\right)$ correspondingly.

Corollary 2. Under the conditions of Proposition 2 there are isomorphisms $B G_{\infty}^{+}$ $\cong \Omega_{0} B\left(\coprod_{n \geq 0} B G_{n}\right)$ and $B H_{\infty}^{+} \cong \Omega_{0} B\left(\coprod_{n \geq 0} B H_{n}\right)$ which fit the commutative diagram analogous to (5).

Proposition 3. There exists an infinite loop space $X$ that fits the following splitting of the infinite loop spaces:

$$
\Omega B\left(\coprod_{n \geq 0} B G_{n}\right) \simeq \Omega^{\infty} S^{\infty} \times X
$$

Proof. We recall results of F. Waldhausen $[\mathrm{W}]$. He studied the space

$$
A(*)=\mathbb{Z} \times \lim _{\rightarrow, k}\left(B \text { Aut }\left(\bigvee^{k} S^{n}\right)\right)^{+}
$$

and proved that the canonical map

$$
B \Sigma_{\infty}^{+} \rightarrow A(*)
$$

splits; that is, there exists a map

$$
A(*) \rightarrow B \Sigma_{\infty}^{+}
$$

such that its composition with the previous one is the homotopy equivalence of $B \Sigma_{\infty}^{+}$. Moreover the maps (6) and (7) are infinite loop maps between the infinite loop spaces (see Remark 5.3 in [W]). A. Hatcher uses this splitting in $[\mathrm{H}]$. He considers the map

$$
\operatorname{Aut}\left(\bigvee^{k} S^{0}\right) \rightarrow \operatorname{Aut}\left(\bigvee^{k} S^{1}\right)
$$

induced by the suspension functor and the composition

$$
\operatorname{Aut}\left(\bigvee^{k} S^{0}\right) \rightarrow \operatorname{Aut}\left(\bigvee^{k} S^{1}\right) \rightarrow \lim _{\rightarrow, k} A u t\left(\bigvee^{k} S^{n}\right)
$$

Then he takes the composition of the map (8) with the Waldhausen map (7) and obtains the analogous splitting for $B A u t_{\infty}^{+}$. We note that Hatcher's arguments are 


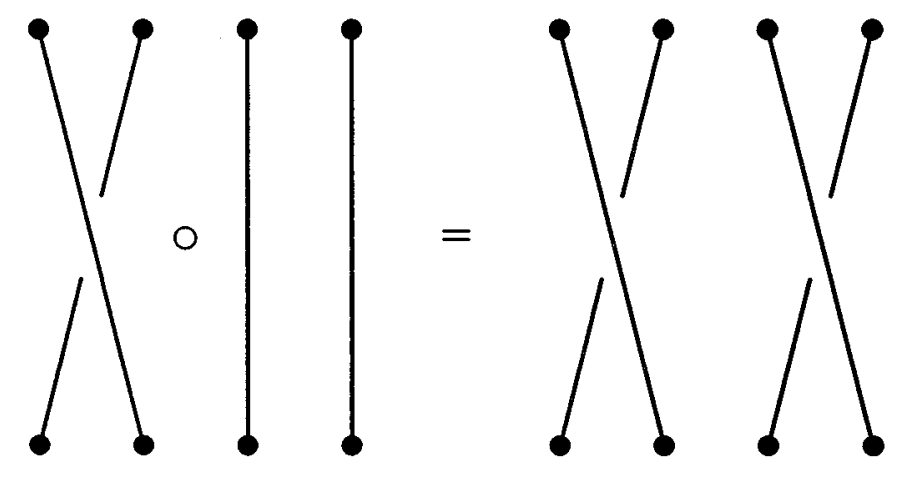

FIGURE 1

valid for our system of subgroups $G_{n}$. Namely, let $X$ be a fibre of the following composition:

$$
\Omega B\left(\coprod_{n \geq 0} B G_{n}\right) \rightarrow \Omega B\left(\coprod_{n \geq 0} B A u t F_{n}\right) \rightarrow \Omega^{\infty} S^{\infty},
$$

where the second map is the Waldhausen map (7). The space $X$ is an infinite loop space, and the infinite loop map

$$
\Omega^{\infty} S^{\infty} \rightarrow \Omega B\left(\coprod_{n \geq 0} B G_{n}\right)
$$

gives the necessary splitting. The proposition is proved.

There is another multiplicative structure for the systems of the symmetric and braid groups:

$$
\begin{gathered}
\Sigma_{k} \times \Sigma_{l} \rightarrow \Sigma_{k l}, \\
B r_{k} \times B r_{l} \rightarrow B r_{k l} .
\end{gathered}
$$

For the symmetric groups this pairing can be described as follows. Let $\sigma \in \Sigma_{k}$ and $s \in \Sigma_{l}$; then $\sigma \circ s \in \Sigma_{k l}$ is given by the formula

$$
\sigma \circ s:(i-1) k+j \mapsto(s(i)-1) k+\sigma(j), \text { for } 1 \leq i \leq l, 1 \leq j \leq k .
$$

This means that $\sigma \circ s$ permutes the $k l$ letters divided into $l$ blocks (with $k$ letters in each) in such a way that it permutes blocks as the permutation $s$ and it permutes letters inside each block as the permutation $\sigma$. The pairing (10) for the braid groups is described in the following way. We thicken each string of the braid $\beta \in B r_{l}$ and embed the braid $\alpha \in B r_{k}$ in each tube. Graphically this is depicted in Figure 1.

Let $\alpha \in A u t F_{k}$ and $\beta \in A u t F_{l}$ be automorphisms of the free groups on letters $x_{1}, \ldots, x_{k}$ and $y_{1}, \ldots, y_{l}$ respectively. We express the action of $\alpha$ and $\beta$ on $x_{j}$ and $y_{k}$ :

$$
\begin{gathered}
\alpha: x_{j} \mapsto \omega_{j}\left(x_{1}, \ldots, x_{k}\right), \\
\beta: y_{i} \mapsto \nu_{i}\left(y_{1}, \ldots, y_{l}\right) .
\end{gathered}
$$


Let $F_{k l}$ be the free group on letters $a_{i, j}$ for $1 \leq i \leq l, 1 \leq j \leq k$. We define an endomorphism $\alpha \circ \beta$ of $F_{k l}$ by the formula

$$
\alpha \circ \beta: a_{i, j} \mapsto \nu_{i}\left(\omega_{j}\left(a_{1,1}, \ldots, a_{1, k}\right), \ldots, \omega_{j}\left(a_{l, 1}, \ldots, a_{l, k}\right)\right) .
$$

Let us consider $A u t F_{k}$ as $\pi_{0}$ of the $H$-space of homotopy equivalences of the wedge of $k$ circles $\bigvee^{k} S^{1}$ : Aut $\left(\bigvee^{k} S^{1}\right)$. The group $A u t F_{k}$ is homotopy equivalent to $\operatorname{Aut}\left(\bigvee^{k} S^{1}\right)$. Then the element $\alpha \circ \beta$ can be described as follows. Let $\beta$ be represented by a map of the bouquet of $l$ circles:

$$
b: \bigvee^{l} S^{1} \rightarrow \bigvee^{l} S^{1}
$$

We mark the points $p_{i, j}, 1 \leq i \leq l, 0 \leq j \leq k$, which divide the $i^{\text {th }}$ circle into $k$ equal parts, with $p_{i, 0}$ the base point of the wedge. We ask of the representative that $b\left(p_{i, j}\right)$ be one of the points $p_{i, j}, 1 \leq i \leq l, 1 \leq j \leq k$. It is possible to do this as follows, for example. We consider the circle which corresponds to the letter $y_{i}$ and let $d_{i}$ be the length of the word $\nu_{i}\left(y_{1}, \ldots, y_{l}\right)$. We map the interval $[0,1]$ linearly onto the interval $\left[0, d_{i}\right]$. Then the points $r / k, r=0,1, \ldots, k$, are mapped to the points $d_{i} r / k$. After factoring by the integral lattice we get the desired representative of $\beta$. This map $b$ defines a homotopy equivalence of $k l$ circles to itself,

$$
b: \bigvee^{k l} S^{1} \rightarrow \bigvee^{k l} S^{1}
$$

The bouquet $\bigvee^{k l} S^{1}$ is naturally divided into $l$ bouquets, having $k$ circles each. Now we apply the homotopy equivalence corresponding to $\alpha$ to each sub-bouquet. We get the homotopy equivalence, corresponding to $\alpha \circ \beta$. So $\alpha \circ \beta$ defined by the formula (11) is an automorphsm of the group $F_{k l}$. This defines a pairing

$$
A u t F_{k} \times A u t F_{l} \rightarrow A u t F_{k l},
$$

compatible with (9) and (10). It is associative. The compatibility with (9) is seen if we interpret the symmetric group the same way as $A u t F_{k}$, putting $S^{0}$ instead $S^{1}$. The map

$$
\operatorname{Aut}\left(\bigvee^{k} S^{0}\right) \rightarrow \operatorname{Aut}\left(\bigvee^{k} S^{1}\right)
$$

is induced by the suspension functor.

Proposition 4. If the system of groups $G_{n}$ is compatible with the pairing (11)(12), then it defines the muliplicative associative (not necessary commutative) spectrum TG with a multiplicative map to the sphere spectrum

$$
T G \rightarrow S,
$$

and this map multiplicatively splits.

Proof. If the system of groups $G_{n}$ from Proposition 1 is compatible with the pairing (12), then the analogous pairing

$$
G_{k} \times G_{l} \rightarrow G_{k l}
$$

is defined. It defines a multiplication of corresponding $\Gamma$-spaces $[S 2, \S 5]$. So the spectrum which corresponds to the infinite loop space $\Omega B\left(\coprod_{n \geq 0} B G_{n}\right)$ is multiplicative $[\mathrm{S} 2, \S 5]$. Let us consider the composition of the pairing (12) with the map 
generated by suspension

$$
\operatorname{Aut}\left(\bigvee^{k} S^{1}\right) \times \operatorname{Aut}\left(\bigvee^{l} S^{1}\right) \rightarrow \operatorname{Aut}\left(\bigvee^{k l} S^{1}\right) \rightarrow \operatorname{Aut}\left(\bigvee^{k l} S^{2}\right)
$$

We get a pairing of $A u t\left(\bigvee^{k} S^{1}\right)$ and $A u t\left(\bigvee^{l} S^{1}\right)$, generated by the smash product. Such a pairing was considered by F. Waldhausen [W] in his study of the space $A(*)$. The canonical maps

$$
\operatorname{Aut}\left(\bigvee^{k} S^{0}\right) \rightarrow \operatorname{Aut}\left(\bigvee^{k} S^{1}\right) \rightarrow \lim _{\rightarrow, k} A u t\left(\bigvee^{k} S^{n}\right)
$$

are compatible with the pairings. It was proved by Waldhausen [W] that the map (7)

$$
A(*) \rightarrow B \Sigma_{\infty}^{+}
$$

is compatible with the multiplicative structures of $A(*)$ and $B \Sigma_{\infty}^{+}$. Proposition 4 is proved.

Looking at the splitting, it is interesting to compare homology of $G_{\infty}$ and $\Sigma_{\infty}$. The answer depends of course on the system of groups $G_{n}$. For example, for the whole group $A u t_{\infty}$ these homologies in dimensions 1 and 2 coincide:

$$
H_{i}\left(A u t_{\infty}, \mathbb{Z}\right)=H_{i}\left(\Sigma_{\infty}, \mathbb{Z}\right) \text { for } i=1,2 .
$$

It was remarked by Hatcher in $[\mathrm{H}]$ that in general (for all $i$ ) it is not known whether this equality holds or not. We shall consider the braid-permutation group in the next section. This is a subgroup of the automorphism group of the free group generated by symmetric and braid groups. We shall see from Theorem 1 below that the case of the braid-permutation is quite different from the case of the whole group $A u t_{\infty}$.

\section{The Braid-Permutation Group}

Let $B P_{n}$ be the subgroup of $A u t F_{n}$ generated by both sets of automorphisms $\xi_{i}$ and $\sigma_{i}$ of (1) and (2). It is called the braid-permutation group. It was proved by R. Fenn, R. Rimányi and C. Rourke in [FRR1], [FRR2] that this group is given by the set of generators: $\left\{\xi_{i}, \sigma_{i}, i=1,2, \ldots, n-1\right\}$ and the relations

$$
\begin{cases}\xi_{i}^{2} & =1, \\ \xi_{i} \xi_{j} & =\xi_{j} \xi_{i}, \text { if }|i-j|>1, \\ \xi_{i} \xi_{i+1} \xi_{i} & =\xi_{i+1} \xi_{i} \xi_{i+1} .\end{cases}
$$

The symmetric group relations

$$
\begin{cases}\sigma_{i} \sigma_{j} & =\sigma_{j} \sigma_{i}, \text { if }|i-j|>1 \\ \sigma_{i} \sigma_{i+1} \sigma_{i} & =\sigma_{i+1} \sigma_{i} \sigma_{i+1}\end{cases}
$$

The braid group relations

$$
\begin{cases}\sigma_{i} \xi_{j} & =\xi_{j} \sigma_{i}, \text { if }|i-j|>1 \\ \xi_{i} \xi_{i+1} \sigma_{i} & =\sigma_{i+1} \xi_{i} \xi_{i+1} \\ \sigma_{i} \sigma_{i+1} \xi_{i} & =\xi_{i+1} \sigma_{i} \sigma_{i+1}\end{cases}
$$

The mixed relations 

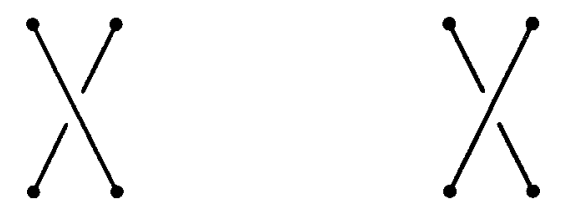

FiguRe 2

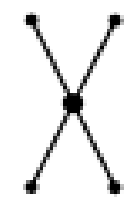

Figure 3

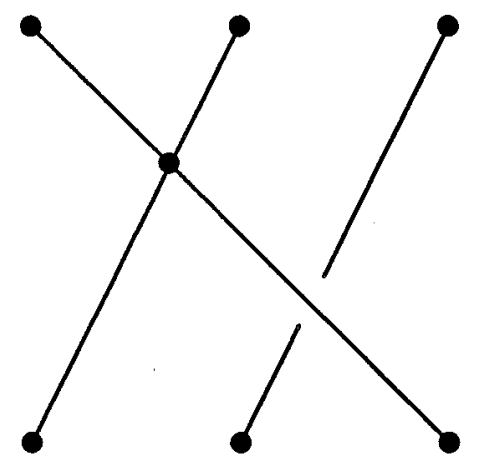

FiguRE 4

Fenn, Rimányi and Rourke also gave the geometrical interpretation of $B P_{n}$ as a group of welded braids. First they define a welded braid diagram on $n$ strings as a collection of $n$ monotone arcs starting from $n$ points on a horizontal line of a plane (the top of the diagram) and going down to $n$ points on another horizontal line (the bottom of the diagram). It is allowed for them to have crossings of two types: 1) as usual braids, as shown in Figure 2, or 2) to have welds having as, shown in Figure 3.

An example of a welded braid diagram is shown in Figure 4.

The composition of welded braid diagrams on $n$ strings is defined by stacking. The diagram with no crossings or welds is an identity with respect to this composition. So the set of welded braid diagrams on $n$ strings forms a semigroup denoted by $W D_{n}$.

Fenn, Rimányi and Rourke defined the following types of allowable moves on welded braid diagrams. They are depicted in Figures 5-8.

The allowable moves shown in Figure 5 are Reidemeister moves known in the knot theory. The first move from Figure 6 corresponds to the relation

$$
\xi_{i}^{2}=1
$$



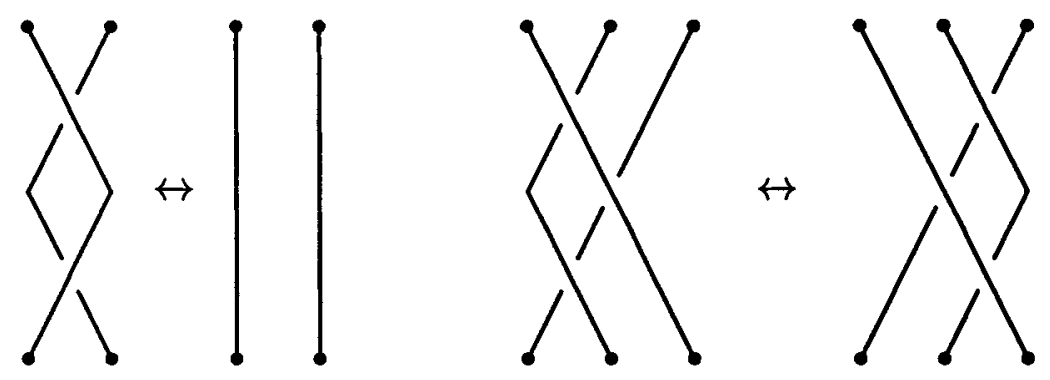

Figure 5
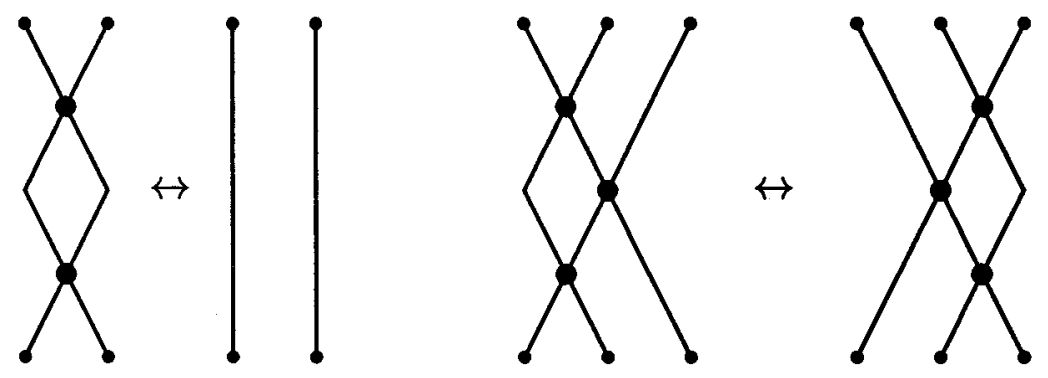

FIGURE 6
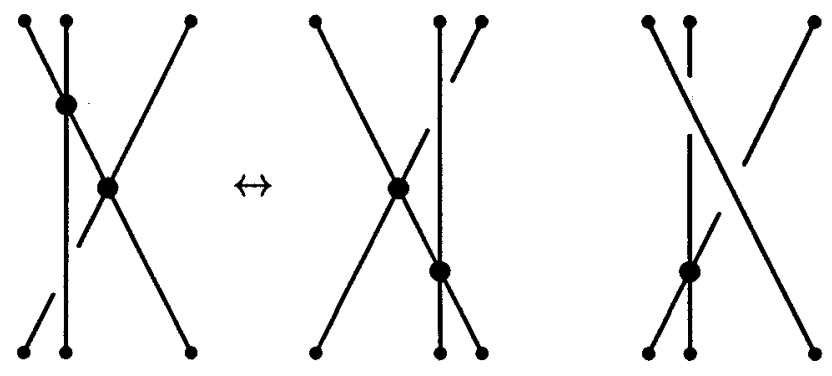

Figure 7

The move from Figure 8 is the geometric form of the commutativity from the mixed relations. There are also analogous moves corresponding to the commutativity from the symmetric group and the braid group relations.

A welded braid is defined as an equivalence class of welded braid diagrams under allowable moves. It was proved by Fenn, Rimányi and Rourke that welded braids form a group, and this group is isomorphic to the braid-permutation group $B P_{n}$. The generator $\sigma_{i}$ corresponds to the canonical generator of the braid group $B r_{n}$ and is shown in Figure 9.

The generators $\xi_{i}$ correspond to the welded braids depicted at the Figure 10.

It is also possible to consider welded braids as objects of a 3-dimensional space. We regard them as imbedded in the positive half (with respect to the third coordinate) of a 3-dimensional space, while the welds are supposed to belong to the 


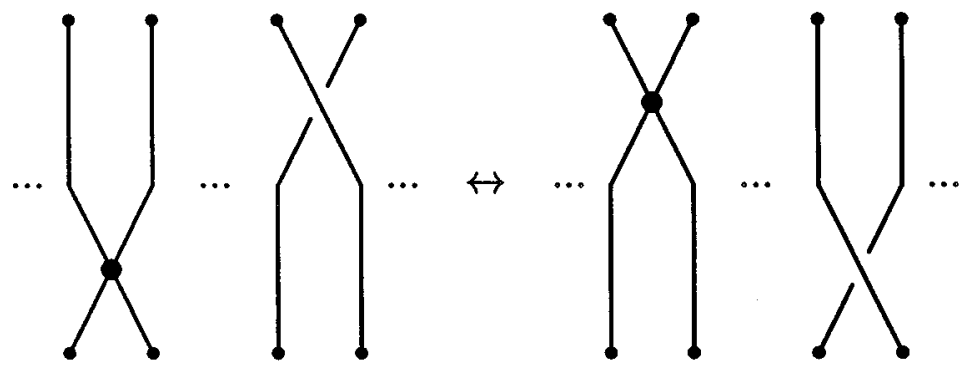

Figure 8

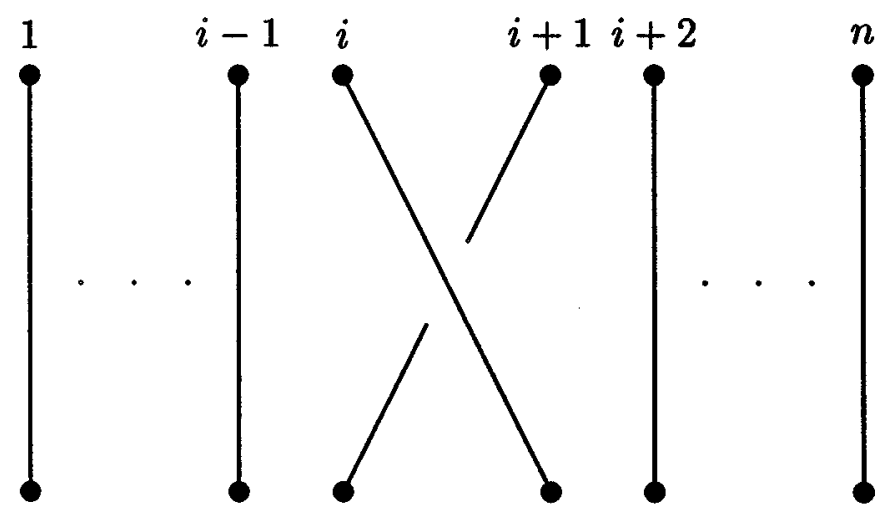

FIGURE 9

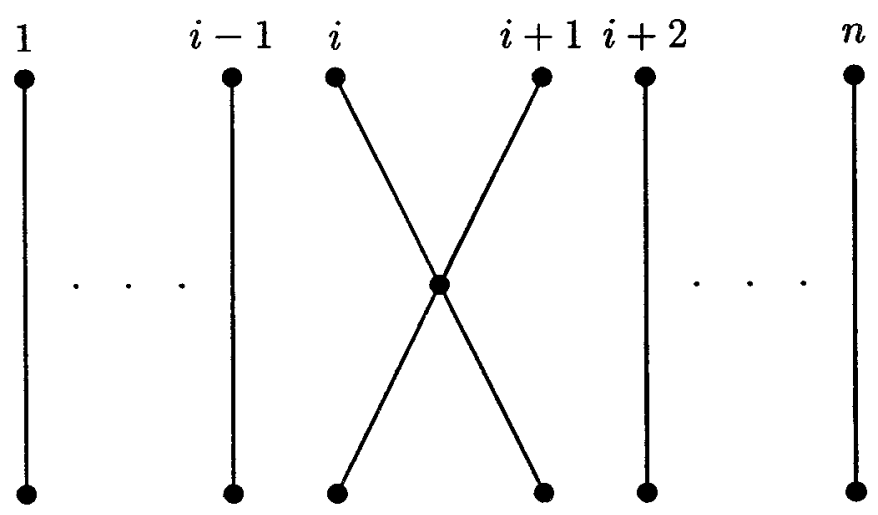

FIGURE 10

two-dimensional plane with the third coordinate equal to zero. Hence strings are not allowed to move behind welds.

The automorphisms of $F_{n}$ which lie in $B P_{n}$ can be characterized in the following way [FRR2]. Let $\pi \in \Sigma_{n}$ be a permutation and $w_{i}, \quad i=1,2, \ldots, n$, be words in $F_{n}$. Then the mapping

$$
x_{i} \mapsto w_{i}^{-1} x_{\pi(i)} w_{i}
$$


determines a homomorphism of $F_{n}$ to itself which is injective. If it is also surjective, then it is called an automorphism of permutation-conjugacy type. The automorphisms of this type determine a subgroup of $A u t F_{n}$ which is precisely $B P_{n}$. From this interpretation we see that the pairing

$$
\mu(n, m): B P_{n} \times B P_{m} \rightarrow B P_{n+m}
$$

and the canonical inclusions

$$
B P_{n} \rightarrow B P_{n+k}
$$

are well defined and satisfy the properties which were considered for the system of groups $G_{m}$. The system of groups $B P_{n}$ is compatible with the pairing (9) for groups of automorphisms of free groups. In fact, let $\alpha \in B P_{k}$ and $\beta \in B P_{l}$ be given by the formulas

$$
\begin{gathered}
\alpha: x_{j} \mapsto w_{j}^{-1} x_{\pi(j)} w_{j}, \\
\beta: y_{i} \mapsto v_{i}^{-1} y_{\sigma(i)} v_{i} .
\end{gathered}
$$

We denote by $z_{p, j}$ the following expression:

$$
w_{j}^{-1}\left(a_{p, 1}, \ldots, a_{p, k}\right) a_{p, \pi(j)} w_{j}\left(a_{p, 1}, \ldots, a_{p, k}\right) .
$$

Then $\alpha \circ \beta \in A u t F_{k l}$ is expressed in the following way:

$$
\begin{aligned}
a_{i, j} \mapsto v_{i}^{-1}\left(z_{1, j}, \ldots, z_{l, j}\right) w_{j}^{-1}\left(a_{\sigma(i), 1}, \ldots, a_{\sigma(i), k}\right) a_{\sigma(i), \pi(j)} & \\
& \times w_{j}\left(a_{\sigma(i), 1}, \ldots, a_{\sigma(i), k}\right) v_{i}\left(z_{1, j}, \ldots, z_{l, j}\right) .
\end{aligned}
$$

So it belongs to the braid-permutation group $B P_{k l}$, and the pairing

$$
B P_{k} \times B P_{l} \rightarrow B P_{k l}
$$

is defined. This pairing defines a multiplication in the $\Gamma$-space corresponding to the system of groups $B P_{n}$.

Let us consider the system of groups $\overline{B P}_{n}$ with the same generators as the braidpermutation group and such that we add two series of relations to the relations of $B P_{n}$ :

$$
\begin{gathered}
\xi_{i} \sigma_{i+1} \sigma_{i}=\sigma_{i+1} \sigma_{i} \xi_{i+1}, \\
\xi_{i} \sigma_{i}=\sigma_{i} \xi_{i} .
\end{gathered}
$$

We call this group the reduced braid-permutation group. So we have the mixed relations for the reduced braid-permutation group in the following form:

$$
\begin{cases}\sigma_{i} \xi_{j} & =\xi_{j} \sigma_{i}, \text { if }|i-j| \neq 1 \\ \xi_{i} \xi_{i+1} \sigma_{i} & =\sigma_{i+1} \xi_{i} \xi_{i+1} \\ \sigma_{i} \sigma_{i+1} \xi_{i} & =\xi_{i+1} \sigma_{i} \sigma_{i+1} \\ \xi_{i} \sigma_{i+1} \sigma_{i} & =\sigma_{i+1} \sigma_{i} \xi_{i+1} .\end{cases}
$$

The mixed relations for $\overline{B P}_{n}$

These considerations lead to the following statement about the classifying spaces of $B P_{\infty}$ and $\overline{B P}_{\infty}$. 
Proposition 5. The canonical epimorphism

$$
B P_{\infty} \rightarrow \overline{B P}_{\infty}
$$

induces a map of the group completions

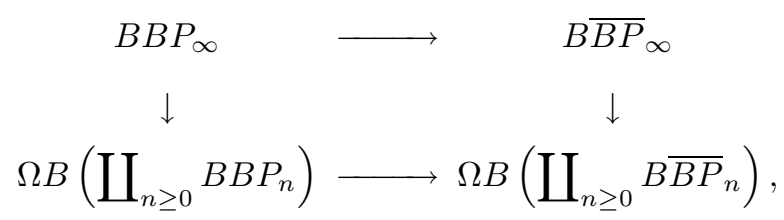

where in the bottom line there is an infinite loop map between the infinite loop spaces. Hence, there are isomorphisms $B B P_{\infty}^{+} \cong \Omega_{0} B\left(\coprod_{n \geq 0} B B P_{n}\right), B \overline{B P}_{\infty}^{+} \cong$ $\Omega_{0} B\left(\coprod_{n \geq 0} B \overline{B P}_{n}\right)$, which fit into the commutative diagram analogous to (13).

We need the following simple fact about the groups $B P_{n}$ and $\overline{B P}_{n}$.

Proposition 6. The abelianizations of $B P_{n}$ and $\overline{B P}_{n}, 2 \leq n \leq \infty$, are both equal to $\mathbb{Z} \oplus \mathbb{Z} / 2$ :

$$
\begin{aligned}
& B P_{n} /\left[B P_{n}, B P_{n}\right] \cong \mathbb{Z} \oplus \mathbb{Z} / 2, \\
& \overline{B P}_{n} /\left[\overline{B P}_{n}, \overline{B P}_{n}\right] \cong \mathbb{Z} \oplus \mathbb{Z} / 2 .
\end{aligned}
$$

Proof. We add the following relations to that of the braid group $B r_{n}$ and symmetric group $\Sigma_{n}$ :

$$
\begin{aligned}
\sigma_{i} & =\sigma_{j} \text { for all } i \text { and } j, \\
\xi_{i} & =\xi_{j} \text { for all } i \text { and } j
\end{aligned}
$$

and obtain the epimorphisms of alelianization for the braid and symmetric groups

$$
a b_{B r}: B r_{n} \rightarrow \mathbb{Z}
$$

and

$$
a b_{\Sigma}: \Sigma_{n} \rightarrow \mathbb{Z} / 2 .
$$

If we add the relations of both types to the defining relations of the braid-permutation group, we get an epimorphism

$$
a b_{B P}: B P_{n} \rightarrow \mathbb{Z} \oplus \mathbb{Z} / 2
$$

On the other hand, if we consider the free product of the braid and symmetric groups $B r_{n} * \Sigma_{n}$, we obtain from the universality that the homomorphism of abelianization for the group $B r_{n} * \Sigma_{n}$ can be defined as the composition

$$
a b_{B P * \Sigma}: B r_{n} * \Sigma_{n} \stackrel{a b_{B r * a b_{\Sigma}}}{\longrightarrow} \mathbb{Z} * \mathbb{Z} / 2 \rightarrow \mathbb{Z} \oplus \mathbb{Z} / 2,
$$

where the second map is the canonical epimorphism. Consider the homomorphism $a b_{B P * \Sigma}$ as the composition

$$
B r_{n} * \Sigma_{n} \rightarrow B P_{n} \stackrel{a b_{B P}}{\longrightarrow} \mathbb{Z} \oplus \mathbb{Z} / 2,
$$

where the first map is the canonical epimorphism. Again using the universality, we see that $a b_{B P}$ is the abelianization of $B P_{n}$. The proof for the reduced braidpermutation group is the same. Proposition 6 is proved. 
There are epimorphisms

$$
\begin{aligned}
& \alpha_{n}: B P_{n} \rightarrow \mathbb{Z}, \\
& \bar{\alpha}_{n}: \overline{B P}_{n} \rightarrow \mathbb{Z}
\end{aligned}
$$

which are given by the formulas

$$
\begin{aligned}
\xi_{i} & \mapsto 0 \text { for all } i, \\
\sigma_{i} & \mapsto 1 \text { for all } i .
\end{aligned}
$$

It follows from the relations that there exists an epimorphism

$$
\phi_{n}: B P_{n} \rightarrow \Sigma_{n}
$$

defined by formulas

$$
\begin{aligned}
\phi_{n}\left(\xi_{i}\right) & =\xi_{i}, \\
\phi_{n}\left(\sigma_{i}\right) & =\xi_{i} .
\end{aligned}
$$

Its composition with the canonical inclusion $\nu_{n}$ of $\Sigma_{n}$ in $B P_{n}$ is equal to the identity map of $\Sigma_{n}$. These homomorphisms generate maps of classifying spaces $B \nu_{n}$ and $B \phi_{n}$ such that their composition

$$
B \Sigma_{n} \rightarrow B B P_{n} \rightarrow B \Sigma_{n}
$$

is equal to the identity. We have also the inclusion $\kappa_{n}$ of the braid group in $B P_{n}$, which generates the map of classifying spaces

$$
B B r_{n} \rightarrow B B P_{n} .
$$

The composition of $\kappa_{n}$ and $\phi_{n}$ gives the canonical epimorphism:

$$
\tau_{n}: B r_{n} \rightarrow \Sigma_{n} .
$$

We denote by $\mathcal{Z}$ a strict monoidal (tensor) category whose objects $\{\overline{0}, \overline{1}, \ldots\}$ correspond to the integer numbers from 0 to infinity, and whose morphisms are defined by the formula

$$
\operatorname{hom}(\bar{k}, \bar{l})=\left\{\begin{array}{lll}
\mathbb{Z}, & \text { if } \quad k=l, \\
\emptyset, & \text { if } \quad k \neq l .
\end{array}\right.
$$

The product in $\mathcal{Z}$ is defined on objects by the sum of nonnegative numbers and on morphisms by the sum of integer numbers. This category has a symmetry which is equal to the zero element for all $\bar{m}$ and $\bar{n}$. The homomorphisms $\alpha_{n}$ induce a morphism of permutative categories

$$
A: \mathcal{B P} \rightarrow \mathcal{Z}
$$

and maps of classifying spaces

$$
B \alpha_{n}: B B P_{n} \rightarrow S^{1} .
$$

We recall that $\gamma$ denotes an inclusion of the group $\mathbb{Z}$ into $B r_{n}$ :

$$
\gamma: \mathbb{Z} \rightarrow B r_{n}
$$

when the generator of the cyclic group is mapped to one of the generators $\sigma_{i}$, say, $\sigma_{1}: \gamma(1)=\sigma_{1}$. 
Theorem 1. There exist maps

$$
\psi: B \Sigma \times B B r \rightarrow B B P^{+}
$$

and

$$
\beta: \Omega B\left(\coprod_{n \geq 0} B B P_{n}\right) \rightarrow \Omega^{\infty} S^{\infty} \times S^{1}
$$

such that the map $\psi$ becomes a loop map after the group completion, the map $\beta$ is an infinite loop map, and it splits by the map

$$
\mathbb{Z} \times B \Sigma^{+} \times S^{1^{I d \times(B \gamma}} \stackrel{+}{\longrightarrow} \mathbb{Z} \times B \Sigma^{+} \times B B r^{+} \stackrel{I d \times \psi^{+}}{\longrightarrow} \mathbb{Z} \times B B P^{+} .
$$

If an infinite loop space $Y$ is a fibre of the map $\beta$, then it fits into the following splitting:

$$
\Omega B\left(\coprod_{n \geq 0} B B P_{n}\right) \simeq \Omega^{\infty} S^{\infty} \times S^{1} \times Y
$$

The same is true for the reduced braid-permutation group with one modification: the map

$$
\bar{\psi}: B \Sigma \times B B r \rightarrow B \overline{B P}^{+}
$$

becomes a double loop map after the group completion.

Proof. The morphism $A$ induces the map of infinite loop spaces

$$
\Omega B\left(\coprod_{n \geq 0} B B P_{n}\right) \rightarrow S^{1}
$$

Analogously the homomorphisms $\phi_{n}$ generate the morphism of the permutative categories

$$
\mathcal{B P} \rightarrow \Sigma
$$

and the corresponding infinite loop map

$$
\Omega B\left(\coprod_{n \geq 0} B B P_{n}\right) \rightarrow \Omega^{\infty} S^{\infty}
$$

We denote by $\beta$ the following composition:

$$
\Omega B\left(\coprod_{n \geq 0} B B P_{n}\right) \stackrel{\text { diag }}{\longrightarrow} \Omega B\left(\coprod_{n \geq 0} B B P_{n}\right) \times \Omega B\left(\coprod_{n \geq 0} B B P_{n}\right) \rightarrow \Omega^{\infty} S^{\infty} \times S^{1} .
$$

The homomorphisms $\nu$ and $\kappa$ induce the map of products of classifying spaces

$$
B \Sigma \times B B r \rightarrow B B P \times B B P .
$$

The space $B B P^{+}$is an $H$-space, so there exists a map

$$
\mu: B B P^{+} \times B B P^{+} \rightarrow B B P^{+} .
$$

Let us consider now the composition $f$ :

$$
B \Sigma \times B B r \stackrel{B \nu \times B \kappa}{\longrightarrow} B B P \times B B P \stackrel{q \times q}{\longrightarrow} B B P^{+} \times B B P^{+} \stackrel{\mu}{\longrightarrow} B B P^{+} .
$$


From the construction we see that the map

$$
B \Sigma \stackrel{\text { equiv }}{\longrightarrow} B \Sigma \times * \stackrel{i d \times \text { incl }}{\longrightarrow} B \Sigma \times B B r \stackrel{f}{\longrightarrow} B B P^{+}
$$

is homotopic to $q B \nu$, and the map

$$
B B r \stackrel{\text { equiv }}{\longrightarrow} * \times B B r \stackrel{\text { incl } \times i d}{\longrightarrow} B \Sigma \times B B r \stackrel{f}{\rightarrow} B B P^{+}
$$

is homotopic to $q B \kappa$. Using the fact that $\mathbb{Z} \times B B P^{+}$is a loop space, we slightly change the map

$$
q B \kappa: B B r \rightarrow B B P^{+},
$$

and define the map

$$
g: B B r \rightarrow B P^{+}
$$

by the formula

$$
g(b)=(q B \tau(b))^{-1} \cdot q B \kappa(b), b \in B B r .
$$

In this case the composition

$$
B^{+} \phi \cdot g: B B r \rightarrow B \Sigma^{+}
$$

is homotopic to zero. We denote by $\psi$ the composition

$$
B \Sigma \times B B r \stackrel{B \nu \times g}{\longrightarrow} B B P \times B B P \stackrel{q \times q}{\longrightarrow} B B P^{+} \times B B P^{+} \stackrel{\mu}{\longrightarrow} B B P^{+}
$$

and by $\chi$ the composition

$$
B \Sigma \times S^{1} \stackrel{\text { identity } \times B \gamma}{\longrightarrow} B \Sigma \times B B r \stackrel{\psi}{\longrightarrow} B B P^{+},
$$

and by $\bar{\psi}$ and $\bar{\chi}$ the corresponding maps for the braid-permutation group. So, the composition

$$
B \Sigma \times B B r \stackrel{\psi}{\longrightarrow} B B P^{+B^{+}} \stackrel{\phi \times B^{+} \alpha}{\longrightarrow} B \Sigma^{+} \times S^{1}
$$

is homotopic to the product $q \times B \alpha$, and the composition

$$
B \Sigma \times S^{1} \stackrel{\chi}{\longrightarrow} B B P^{+} B^{+} \stackrel{\phi \times B^{+} \alpha}{\longrightarrow} B \Sigma^{+} \times S^{1}
$$

is the canonical map from a space to its plus-construction. It gives the following splitting:

$$
B \Sigma^{+} \times S^{1} \stackrel{\chi^{+}}{\longrightarrow} B B P^{+} B^{+} \stackrel{\phi \times B^{+}}{\longrightarrow} B \Sigma^{+} \times S^{1} .
$$

The map $B \kappa$ fits into the commutative diagram

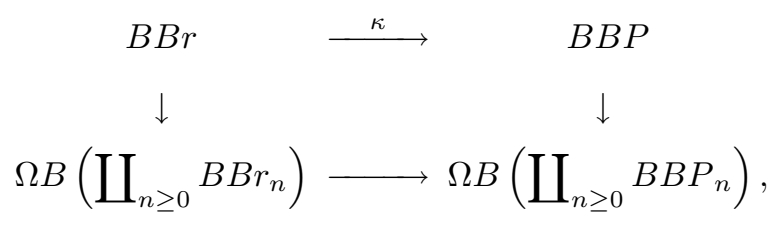

where in the bottom line one has a loop map.

The category $\mathcal{B}$, generated by the braid groups, is a braided category as defined by A. Joyal and R. Street [JS]. This means that there is a system of isomorphisms of objects

$$
c_{\bar{m}, \bar{n}}: \overline{m+n} \rightarrow \overline{m+n}
$$




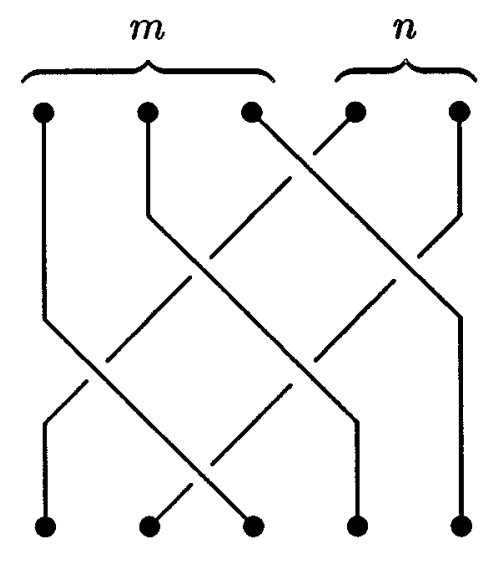

FIGURE 11

natural with respect to morphisms from $\bar{m}$ to itself and from $\bar{n}$ to itself and satisfying the properties of coherence B1 and B2 from [JS], which are sometimes called the Drinfeld triangles. A braided category becomes a symmetric monoidal category if its braiding satisfies the additional property (5). We consider as the braiding $c$ in $\mathcal{B}$ the following system of elements:

$$
\sigma_{m} \ldots \sigma_{1} \sigma_{m+1} \ldots \sigma_{2} \ldots \sigma_{n+m-1} \ldots \sigma_{n} \in B r_{m+n}
$$

Graphically this is shown in Figure 11.

The image of the braiding $c$ by the functor

$$
\overline{\mathcal{K}}: \mathcal{B} \rightarrow \overline{\mathcal{B P}}
$$

is a braiding in the category $\overline{\mathcal{B P}}$. The proof is the same as the proof of Proposition 7 in the next section.

Modifying P. May's proof [May] of his theorem that the group completion of the classifying space of a symmetric monoidal category is an infinite loop space, to the case of braided category, we can prove the analogous fact in this case. Namely, the group completion of the classifying space of a braided monoidal category is a double loop space and a morphism of braided categories induces a double loop map. This was done recently by Z. Fiedorowicz [Fi] and M. Batanin [Bat]. This means that in our case the map of classifying spaces

$$
B \mathcal{B} \rightarrow B \overline{\mathcal{B P}}
$$

induced by $\overline{\mathcal{K}}$, becomes a double loop map after group completion. Theorem 1 is proved.

Remark. It is not asserted that the splitting of Theorem 1 is a splitting of infinite loop spaces.

Corollary 3. In homologies with coefficients in $\mathbb{Z}$ the epimorphism of algebras

$$
\beta_{*}: H_{*}(B P ; \mathbb{Z}) \rightarrow H_{*}(\Sigma \times \mathbb{Z} ; \mathbb{Z})
$$

splits (as a morphism of abelian groups) by the monomorphism

$$
\chi_{*}: H_{*}(\Sigma \times \mathbb{Z} ; \mathbb{Z}) \rightarrow H_{*}(B P ; \mathbb{Z}) .
$$


The homologies with coefficients in $\mathbb{Z} / p$ of the infinite braid-permutation group $H_{*}(B P, \mathbb{Z} / p)$ as an $H_{*}(\Sigma ; \mathbb{Z} / p)$-module are isomorphic to

$$
H_{*}(\Sigma ; \mathbb{Z} / p) \otimes H_{*}(\mathbb{Z} ; \mathbb{Z} / p) \otimes H_{*}(Y ; \mathbb{Z} / p) .
$$

The same is true for the homology of the reduced braid-permutation group $\overline{B P}$.

The homomorphism of the inclusion

$$
B P_{n} \rightarrow A u t F_{n}
$$

generates an $E_{\infty}$-map of classifying spaces of the corresponding categories

$$
B \mathcal{B P} \rightarrow B \mathcal{A} u t
$$

and the corresponding map of the infinite loop spaces

$$
\Omega B\left(\coprod_{n \geq 0} B B P_{n}\right) \rightarrow \Omega B\left(\coprod_{n \geq 0} B A u t_{n}\right) .
$$

If we consider the composition

$$
B \Sigma^{+} \times \mathrm{BBr}^{+} \rightarrow \mathrm{BBP}^{+} \rightarrow\left(B A u t_{\infty}\right)^{+},
$$

we see that in $\bmod p$ homology the elements of $H_{*}(B P, \mathbb{Z} / p)$ which are images of elements of $H_{*}(B r, \mathbb{Z} / p)$ and those which are obtained from them by ArakiKudo-Dayer-Lashof operations [CLM] are mapped to the $H_{*}(B \Sigma, \mathbb{Z} / p)$ part of the homology of $A u t_{\infty}$.

Let $\pi_{i}^{S}, i=0,1, \ldots$, denote the stable homotopy groups of spheres. The infinite loop spaces $\mathbb{Z} \times B B P^{+}$and $\mathbb{Z} \times B \overline{B P}^{+}$determine generalized homology theories, which we shall denote by $P B_{*}(\cdot)$ (in order not to be confused with Brown-Peterson theory) and $\overline{P B}_{*}(\cdot)$. Our considerations also prove the following proposition.

Corollary 4. The series of braid-permutation groups defines a generalized multiplicative homology theory $P B_{*}(\cdot)$, whose groups of coefficients $P B_{i}$ contain as a direct summand $\pi_{i}^{S} \oplus \pi_{i}\left(S^{1}\right)$.

\section{The Baez-Birman monoid}

The Baez-Birman monoid $S B_{n}$ (or generalized braid monoid, or singular braid monoid) [Bae], [Bi] is defined as the monoid with generators $g_{i}, g_{i}^{-1}, a_{i}, i=1, \ldots$, $n-1$, and relations

$$
\begin{aligned}
& g_{i} g_{j}=g_{j} g_{i}, \text { if }|i-j|>1, \\
& a_{i} a_{j}=a_{j} a_{i}, \text { if }|i-j|>1, \\
& a_{i} g_{j}=g_{j} a_{i}, \text { if }|i-j| \neq 1, \\
& g_{i} g_{i+1} g_{i}=g_{i+1} g_{i} g_{i+1}, \\
& g_{i} g_{i+1} a_{i}=a_{i+1} g_{i} g_{i+1}, \\
& g_{i+1} g_{i} a_{i+1}=a_{i} g_{i+1} g_{i}, \\
& g_{i} g_{i}^{-1}=g_{i}^{-1} g_{i}=1 .
\end{aligned}
$$

In pictures $g_{i}$ corresponds to a canonical generator of the braid group (right-handed crossing) and $a_{i}$ represents an intersection of the $i$ th and $(i+1)$ st strand, just as in Figures 9 and 10. A more detailed geometric interpretation of the Baez-Birman monoid can be found in Joan Birman's paper [Bi]. 
A homomorphism $j_{n}$ from the braid group $B r_{n}$ is evidently defined:

$$
j_{n}: B r_{n} \rightarrow S B_{n} .
$$

It is proved by R. Fenn, E. Keyman and C. Rourke [FKR] that the Baez-Birman monoid embeds in a group $S G_{n}$ which they call the singular braid group:

$$
S B_{n} \rightarrow S G_{n} \text {. }
$$

This means that the elements $a_{i}$ become invertible and all the relations of $S B_{n}$ remain true. The pairings

$$
\begin{gathered}
\mu_{m, n}: S B_{m} \times S B_{n} \rightarrow S B_{m+n}, \\
\mu_{m, n}: S G_{m} \times S G_{n} \rightarrow S G_{m+n}
\end{gathered}
$$

are defined in a usual way by the formulas

$$
\begin{gathered}
\mu_{m, n}\left(g_{i}^{\prime}\right)=g_{i}, \mu_{m, n}\left(a_{i}^{\prime}\right)=a_{i} ; \\
g_{i}^{\prime}, a_{i}^{\prime} \in S B_{m}, S G_{m} ; g_{i}, a_{i} \in S B_{m+n}, S G_{m+n} \\
\mu_{m, n}\left(g_{j}^{\prime \prime}\right)=g_{j+m}, \mu_{m, n}\left(a_{j}^{\prime \prime}\right)=a_{j+m} ; \\
g_{i}^{\prime \prime}, a_{i}^{\prime \prime} \in S B_{n}, S G_{n} ; g_{j+m}, a_{j+m} \in S B_{m+n}, S G_{m+n} .
\end{gathered}
$$

These pairings agree with the corresponding pairing for the braid groups. They define strict monoidal categories $\mathcal{S B}$ and $\mathcal{S G}$, and homomorphisms $j_{n}$ and the canonical inclusion $S B_{n} \rightarrow S G_{n}$ define functors from the strict monoidal category $\mathcal{B}$ generated by the braid groups:

$$
\begin{gathered}
\mathcal{J}: \mathcal{B} \rightarrow \mathcal{S B}, \\
\mathcal{B} \stackrel{\mathcal{J}}{\longrightarrow} \mathcal{S B} \rightarrow \mathcal{S G},
\end{gathered}
$$

which are morphisms of monoidal categories. The pairings $\mu$ are commutative up to conjugation, just as for the braid groups. More precisely, we have the following proposition.

Proposition 7. The image of the braiding $c$ in the category $\mathcal{B}$ by the functor $\mathcal{J}$ is a braiding in the category $\mathcal{S B}$, so it becomes a braided category and the functor $\mathcal{J}$ becomes a morphism between the braided categories. In the same way $\mathcal{S G}$ becomes a braided category, and the corresponding functor becomes a morphism between the braided categories.

Proof. We prove the proposition for $\mathcal{S B}$. The proof for $\mathcal{S G}$ is the same. By definition, the naturality of the braiding $\mathcal{J}(c)$ (which we shall denote by the same symbol c) means that

$$
c_{\bar{m}, \bar{n}} \cdot \mu\left(b_{m}^{\prime}, b_{n}^{\prime \prime}\right)=\mu\left(b_{n}^{\prime \prime}, b_{m}^{\prime}\right) \cdot c_{\bar{m}, \bar{n}} .
$$

This is equivalent to the expression

$$
c_{\bar{m}, \bar{n}} \cdot \mu\left(b_{m}^{\prime}, b_{n}^{\prime \prime}\right) \cdot c_{\bar{m}, \bar{n}}^{-1}=\mu\left(b_{n}^{\prime \prime}, b_{m}^{\prime}\right),
$$

which means that conjugation by the element $c_{\bar{m}, \bar{n}}$ transforms the elements of $S B_{m} \times S B_{n}$, canonically lying in $S B_{m+n}$, into the corresponding elements of $S B_{n} \times$ $S B_{m}$. The elements $c_{\bar{m}, \bar{n}}$ define a braiding for the category $\mathcal{B}$, so, for checking 
the naturality of $c$ in $\mathcal{S B}$, it remains to verify the naturality for the generators $a_{i}, 1 \leq i \leq m-1, m \leq i \leq m+n$. Let us consider the corresponding conjugation:

$$
g_{m} \ldots g_{1} g_{m+1} \ldots g_{2} \ldots g_{n+m-1} \ldots g_{n} a_{i} g_{n}^{-1} \ldots g_{n+m-1}^{-1} \ldots g_{2}^{-1} g_{m+1}^{-1} g_{1}^{-1} \ldots g_{m}^{-1} \text {. }
$$

If $i>n$, we move $a_{i}$ back, using the relation

$$
a_{i} g_{i+1} g_{i}=g_{i+1} g_{i} a_{i+1} .
$$

We have

$$
\begin{gathered}
g_{m} \ldots g_{1} g_{m+1} \ldots g_{2} \ldots g_{n+m-1} \ldots g_{n} a_{i} g_{n}^{-1} \ldots g_{n+m-1}^{-1} \ldots g_{2}^{-1} g_{m+1}^{-1} g_{1}^{-1} \ldots g_{m}^{-1} \\
=g_{m} \ldots g_{1} g_{m+1} \ldots g_{2} \ldots g_{n+m-1} \ldots g_{i-1} a_{i} g_{i-1}^{-1} \ldots g_{n+m-1}^{-1} \ldots g_{2}^{-1} g_{m+1}^{-1} g_{1}^{-1} \ldots g_{m}^{-1} \\
=g_{m} \ldots g_{1} g_{m+1} \ldots g_{2} \ldots g_{n+m-1} \ldots g_{i+1} a_{i-1} g_{i} g_{i-1} g_{i-1}^{-1} \ldots g_{n+m-1}^{-1} \ldots g_{2}^{-1} g_{m+1}^{-1} g_{1}^{-1} \ldots g_{m}^{-1} \\
=\ldots=a_{i-n} .
\end{gathered}
$$

If $i<n$, we move $a_{i}$ back, using the relation

$$
a_{i+1} g_{i} g_{i+1}=g_{i} g_{i+} a_{i} .
$$

We have

$$
\begin{gathered}
g_{m} \ldots g_{1} g_{m+1} \ldots g_{2} \ldots g_{n+m-1} \ldots g_{n} a_{i} g_{n}^{-1} \ldots g_{n+m-1}^{-1} \ldots g_{2}^{-1} g_{m+1}^{-1} g_{1}^{-1} \ldots g_{m}^{-1} \\
=g_{m} \ldots g_{1} g_{m+1} \ldots g_{2} \ldots g_{i} g_{n+m-1} \ldots g_{i+2} g_{i+1} a_{i} g_{i+1}^{-1} \ldots g_{n+m-1}^{-1} \ldots g_{2}^{-1} g_{m+1}^{-1} g_{1}^{-1} \ldots g_{m}^{-1} \\
=g_{m} \ldots g_{1} g_{m+1} \ldots g_{2} \ldots g_{i} g_{n+m-1} \ldots g_{i+2} g_{i}^{-1} g_{i} g_{i+1} a_{i} \\
\times g_{i+1}^{-1} \ldots g_{n+m-1}^{-1} g_{i}^{-1} \ldots g_{2}^{-1} g_{m+1}^{-1} g_{1}^{-1} \ldots g_{m}^{-1} \\
=g_{m} \ldots g_{1} g_{m+1} \ldots g_{2} \ldots g_{i+1} g_{n+m-1} \ldots g_{i+2} a_{i+1} \\
\times g_{i} g_{i+1} g_{i+1}^{-1} \ldots g_{n+m-1}^{-1} g_{i}^{-1} \ldots g_{2}^{-1} g_{m+1}^{-1} g_{1}^{-1} \ldots g_{m}^{-1} \\
=g_{m} \ldots g_{1} g_{m+1} \ldots g_{2} \ldots g_{i+1} g_{n+m-1} \ldots g_{i+2} a_{i+1} g_{i+2}^{-1} \ldots g_{n+m-1}^{-1} g_{i+1}^{-1} \ldots g_{2}^{-1} g_{m+1}^{-1} g_{1}^{-1} \ldots g_{m}^{-1} \\
=\ldots=a_{i+m} .
\end{gathered}
$$

The conditions of coherence are fulfilled trivially. For condition B1 of [JS] we have identically

$$
\begin{gathered}
g_{m} \ldots g_{1} g_{m+1} \ldots g_{2} \ldots g_{n+m-1} \ldots g_{n} \cdot g_{m+n} \ldots g_{n+1} g_{m+n+1} \ldots g_{n+2} \ldots g_{n+m+q-1} \ldots g_{n+q} \\
=g_{m} \ldots g_{1} g_{m+1} \ldots g_{2} \ldots g_{n+m+q-1} \ldots g_{n+q} .
\end{gathered}
$$

For B2 it is also evident:

$$
\begin{gathered}
g_{m+n} \ldots g_{m+1} g_{m+n+1} \ldots g_{m+2} \ldots g_{n+m+q-1} \ldots g_{m+q} \cdot g_{m} \ldots g_{1} g_{m+1} \ldots g_{2} \ldots g_{m+q-1} \ldots g_{q} \\
=g_{m+n} \ldots g_{1} g_{m+n+1} \ldots g_{2} \ldots g_{m+n+q-1} \ldots g_{q} .
\end{gathered}
$$

Proposition 7 is proved. 
Let $B S B$ and $B S G$ denote the classifying spaces of the infinite Baez-Birman monoid and the corresponding singular braid group. The pairings $\mu_{m, n}$ define, as usual, a monoid structure on the disjoint sum of the classifying spaces of $S B_{n}$ and $S G_{n}$ :

$$
\coprod_{n \geq 0} B S B_{n}, \quad \coprod_{n \geq 0} B S G_{n}
$$

Proposition 8. The canonical maps

$$
B S B \rightarrow \Omega B\left(\coprod_{n \geq 0} B S B_{n}\right)
$$

and

$$
B S G \rightarrow \Omega B\left(\coprod_{n \geq 0} B S G_{n}\right)
$$

induce isomorphisms in homology

$$
\begin{aligned}
& H_{*}(B S B ; A) \rightarrow H_{*}\left(\left(\Omega B\left(\coprod_{n \geq 0} B S B_{n}\right)\right)_{0} ; A\right), \\
& H_{*}(B S G ; A) \rightarrow H_{*}\left(\left(\Omega B\left(\coprod_{n \geq 0} B S G_{n}\right)\right)_{0} ; A\right),
\end{aligned}
$$

with any (constant) coefficients. So,

$$
\begin{aligned}
& B S B^{+} \cong\left(\Omega B\left(\coprod_{n \geq 0} B S B_{n}\right)\right)_{0}, \\
& B S G^{+} \cong\left(\Omega B\left(\coprod_{n \geq 0} B S G_{n}\right)\right)_{0} .
\end{aligned}
$$

The proof is the same as the proof of the Theorem 3.2.1 and Corollary 3.2.2 in [Ad] or (what is essentially the same) directly based on [May]. The braiding $c$ gives the necessary homotopy commutativity for the $H$-spaces $\coprod_{n \geq 0} B S B_{n}$ and $\coprod_{n \geq 0} B S G_{n}$.

Comparing the relations of the Baez-Birman monoid and the reduced braidpermutation group, we define the evident homomorphism $l_{n}$ by the formulas

$$
\begin{aligned}
& l_{n}\left(g_{i}\right)=\sigma_{i}, \\
& l_{n}\left(a_{i}\right)=\xi_{i} .
\end{aligned}
$$

The same formulas define the homomorphism

$$
S G_{n} \rightarrow \overline{B P}_{n} .
$$

The composition $l_{n} \circ j_{n}$ is equal to the canonical homomorphism

$$
\kappa_{n}: B r_{n} \rightarrow \overline{B P}_{n} .
$$


The homomorphisms $l_{n}, n=0,1 \ldots$, define a morphism of monoidal categories

$$
\mathcal{L}: \mathcal{S B} \rightarrow \mathcal{S G} \rightarrow \overline{\mathcal{B P}}
$$

such that the image of the braiding $c$ is a braiding in the category $\overline{\mathcal{B P}}$.

Theorem 2. The homomorphisms $j_{n}$ and $l_{n}$ induce morphisms of braided monoidal categories

$$
\mathcal{B} \stackrel{\mathcal{J}}{\longrightarrow} \mathcal{S B} \longrightarrow \mathcal{S G} \stackrel{\mathcal{L}}{\longrightarrow} \overline{\mathcal{B P}}
$$

and the corresponding double loop maps

$$
\Omega^{2} S^{2} \longrightarrow \Omega B\left(\coprod_{n \geq 0} B S B_{n}\right) \longrightarrow \Omega B\left(\coprod_{n \geq 0} B S G_{n}\right) \longrightarrow \Omega B\left(\coprod_{n \geq 0} B \overline{B P}_{n}\right)
$$

Proof. This follows from the fact that the classifying space of a braided monoidal category is an double loop space.

Proposition 9. The abelianization of $S G_{n}$ is equal to $\mathbb{Z} \oplus \mathbb{Z}$ :

$$
S G_{n} /\left[S G_{n}, S G_{n}\right] \cong \mathbb{Z} \oplus \mathbb{Z}
$$

Proof. We add the following relations to that of the singular braid group $S G_{n}$ :

$$
\begin{aligned}
& g_{i}=g_{j} \text { for all } i \text { and } j, \\
& a_{i}=a_{j} \text { for all } i \text { and } j,
\end{aligned}
$$

and obtain an epimorphism

$$
a b_{S B}: S B_{n} \rightarrow \mathbb{Z} \oplus \mathbb{Z}
$$

Let us denote by $g$ and $a$ the generators of $\mathbb{Z} \oplus \mathbb{Z}$, which are the images of the corresponding generators of $S G_{n}$. Suppose that we have a homomorphism to an abelian group $A$ :

$$
f: S G_{n} \rightarrow A .
$$

It follows from the relations of the singular braid group $S G_{n}$ that $f\left(g_{i}\right)=f\left(g_{j}\right)$ and $f\left(a_{i}\right)=f\left(a_{j}\right)$ for all $i$ and $j$. Hence the homomorphism

$$
f^{\prime}: \mathbb{Z} \oplus \mathbb{Z} \rightarrow A
$$

is uniquely determined by the formulas

$$
\begin{aligned}
& f^{\prime}(g)=f\left(g_{i}\right), \\
& f^{\prime}(a)=f\left(a_{i}\right) .
\end{aligned}
$$

This means that the epimorphism $a b_{S B}$ provides the abelianization of $S G_{n}$. Proposition 9 is proved.

There exist maps which are right inverses of the homomorphism

$$
j_{n}: B r_{n} \rightarrow S G_{n}
$$

We define one of them:

$$
h_{n}: S G_{n} \rightarrow B r_{n},
$$

by the formulas

$$
h_{n}\left(g_{i}\right)=\sigma_{i},
$$




$$
h_{n}\left(a_{i}\right)=e,
$$

and another one,

$$
h_{n}^{\prime}: S G_{n} \rightarrow B r_{n}
$$

by the following action on generators:

$$
\begin{aligned}
& h_{n}^{\prime}\left(g_{i}\right)=\sigma_{i}, \\
& h_{n}^{\prime}\left(a_{i}\right)=\sigma_{i} .
\end{aligned}
$$

Let $\operatorname{deg}_{n}$ be the homomorphism

$$
S G_{n} \rightarrow \mathbb{Z}
$$

which assigns to each element of the group $S G_{n}$ the sum of degrees of the generators $a_{i}$ which occur in this element. Let us define the braiding

$$
c_{\bar{m}, \bar{n}}: \overline{m+n} \rightarrow \overline{m+n}
$$

in the category $\mathcal{Z}: c_{\bar{m}, \bar{n}}=m \cdot n$. Then the homomorphisms $\operatorname{deg}_{n}$ generate a functor of the braided categories

$$
\mathcal{S G} \rightarrow \mathcal{Z}
$$

and the corresponding double loop map

$$
\Omega B\left(\coprod_{n \geq 0} B S G_{n}\right) \rightarrow S^{1} .
$$

Analogously the homomorphisms $h_{n}$ generate the functor of the braided categories

$$
\mathcal{S G} \rightarrow \mathcal{B}
$$

and the corresponding double loop map

$$
\Omega B\left(\coprod_{n \geq 0} B S G_{n}\right) \rightarrow \Omega^{2} S^{2}
$$

We denote by $\lambda$ the following composition:

$$
\Omega B\left(\coprod_{n \geq 0} B S G_{n}\right) \stackrel{\operatorname{diag}}{\longrightarrow} \Omega B\left(\coprod_{n \geq 0} B S G_{n}\right) \times \Omega B\left(\coprod_{n \geq 0} B S G_{n}\right) \rightarrow S^{1} \times \Omega^{2} S^{2} .
$$

Let $W$ be a double loop space which is a fibre of the map $\lambda$. One defines a homomorphism

$$
\mathbb{Z} \rightarrow S G_{n}
$$

as an inclusion of the cyclic group as a subgroup generated by the element $a_{1}$. Then the composition

$$
\mathbb{Z} \rightarrow S G_{n} \stackrel{\operatorname{deg}_{n}}{\longrightarrow} \mathbb{Z}
$$

is the identity. Just as in the previous section, we construct a map

$$
\psi_{S}: S^{1} \times B B r \rightarrow B S G^{+} .
$$


Theorem 3. The double loop map

$$
\lambda: \Omega B\left(\coprod_{n \geq 0} B S G_{n}\right) \rightarrow S^{1} \times \Omega^{2} S^{2}
$$

splits by a loop map

$$
1 \times \psi_{S}^{+}: \mathbb{Z} \times S^{1} \times B B r^{+} \rightarrow \mathbb{Z} \times B S G^{+} .
$$

So one has an equivalence of loop spaces

$$
\mathbb{Z} \times B S G^{+} \simeq S^{1} \times \Omega^{2} S^{2} \times W
$$

The proof is analogous to that of the corresponding statement of Theorem 1 .

Corollary 5. In homologies with coefficients in $\mathbb{Z}$ the epimorphism of algebras

$$
\lambda_{*}: H_{*}(S G ; \mathbb{Z}) \rightarrow H_{*}(\mathbb{Z} \times B r ; \mathbb{Z})
$$

splits by the monomorphism

$$
\psi_{S_{*}}: H_{*}(\mathbb{Z} \times B r ; \mathbb{Z}) \rightarrow H_{*}(S G ; \mathbb{Z}) .
$$

The homologies with coefficients in $\mathbb{Z} / p$ of the infinite singular braid group $H_{*}(S G ; \mathbb{Z} / p)$ are isomorphic to $H_{*}(\mathbb{Z} ; \mathbb{Z} / p) \otimes H_{*}(B r ; \mathbb{Z} / p) \otimes H_{*}(W ; \mathbb{Z} / p)$ as algebras.

\section{ACKNOWLEDGEMENTS}

This work was done when the author was holding the position PAST at the University of Nantes. He is thankful to the Department of Mathematics of the University of Nantes and its Geometry-Topology group for the hospitality and the stimulating research atmosphere. Also the author would like to thank Roger Fenn for giving the preprints of the papers [FRR2], [FKR], Hans-Werner Henn and Ulrike Tillmann for discussions on the material of this paper, Haynes Miller for critical remarks and advice on the text of the paper, and the referee for many suggestions of improvements in the exposition of the paper.

\section{REFERENCES}

[Ad] J. F. Adams, Infinite loop spaces, Ann. Math. Stud., No 90, Princeton Univ. Press and Univ. of Tokyo Press, 1978. MR 80d:55001

[Arn1] V. I. Arnold, On some topological invariants of algebraic functions, Trudy Moskov. Mat. Obshch. 21 (1970), 27-46 (Russian), English transl. in Trans. Moscow Math. Soc. 21 (1970), 30-52. MR 43:225

[Arn2] V. I. Arnold, Topological invariants of algebraic functions II, Funk. Anal. i Prilozhen. 4, No 2 (1970), 1-9 (Russian), English transl. in Functional Anal. Appl. 4 (1970), 91-98. MR 43:1991

[Art1] E. Artin, Theorie der Zopfe, Abh. Math. Semin. Univ. Hamburg 4 (1925), 47-72.

[Art2] E. Artin, Theory of braids, Ann. of Math. 48 (1947), 101-126. MR 8:367a

[Bae] John C. Baez, Link invariants of finite type and perturbation theory, Letters in Math. Physics 26 (1992), 43-51. MR 93k:57006

[Bar] M. G. Barratt, A free group functor for stable homotopy, Algebraic Topology (Proc. of Symposia in Pure Math. No. 22) AMS, Providence, 1971, 31-35. MR 48:3043

[Bat] M. Batanin, Private communication.

[Bi] Joan S. Birman, New points of view in knot theory, Bull. of the Amer. Math. Soc. 28 (1993), 253-387. MR 94b:57007

[CF1] F. Cohen, Cohomology of braid spaces, Bull. of the Amer. Math. Soc. 79 (1973), 763-766. MR 47:9607 
[CF2] F. Cohen, Homology of $\Omega^{n+1} \Sigma^{n+1} X$ and $C_{n+1} X, n>0$, Bull. of the Amer. Math. Soc. 79 (1973), 1236-1241. MR 49:3939

[CF3] F. Cohen, Braid orientations and bundles with flat connections, Inventiones Math. 46 (1978), 99-110. MR 80b:57033

[CLM] F. Cohen, T. Lada and J. P. May, The homology of iterated loop spaces, (Lecture Notes in Math.; No 533), Springer-Verlag, Berlin a. o., 1976. MR 55:9096

[FKR] R. Fenn, E. Keyman and C. Rourke, The singular braid monoid embeds in a group, Preprint, 1996

[FRR1] R. Fenn, R. Rimányi and C. Rourke, Some remarks on the braid-permutation group, Topics in Knot Theory. Kluwer Academic Publishers, 1993, 57-68. MR 95g:57022

[FRR2] R. Fenn, R. Rimányi and C. Rourke, The Braid-Permutation Group, Topology 36 (1997), 123-135. MR 97g:20041

[Fi] Z. Fiedorowicz, Operads and iterated monoidal categories, Preprint (1995).

[Fuks] D. B. Fuks, Quillenization and bordisms, Funkcional. Anal. i Prilozh. 8, No 1 (1974), 36-42 (Russian), English transl. in Functional Anal. Appl. 8 (1974), 31-36. MR 49:8043

$[\mathrm{H}] \quad$ Allen Hatcher, Homological stability for automorphism groups of free groups, Comment. Math. Helvetici 70 (1995), 39-62. MR 95k:20030

[J] V. F. R. Jones, Hecke algebra representations of braid groups and link polynomials, Ann. of Math. 126 (1987), 335-388. MR 89c:46092

[JS] A. Joyal and R. Street, Braided tensor categories, Advances in Math. 102 (1993), 20-78. MR 94m: 18008

[ML] S. Mac Lane, Categories for the Working Mathematician, Graduate Texts in Mathematics, Springer-Verlag, N.Y. a.o., 1971. MR 50:7275

[Mah1] M. Mahowald, A new infinite family in ${ }_{2} \pi_{*}^{s}$, Topology 16 (1977), 249-256. MR 56:3838

[Mah2] M. Mahowald, Ring spectra which are Thom complexes, Duke Math. J. 46 (1979), 549559. MR 81f:55010

[May] J. P. May, $E_{\infty}$ spaces, group completions, and permutative categories, New Developments in Topology (London Math. Soc. Lecture Notes Series; No 11) Cambridge, 1974, 61-93. MR 49:3915

[P] Stewart B. Priddy, On $\Omega^{\infty} S^{\infty}$ and the infinite symmetric group, Algebraic Topology (Proc. of Symposia in Pure Math.; No 22) AMS, Providence, 1971, 217-220. MR 50:11226

[S1] G. Segal, Configuration spaces and iterated loop spaces, Inventiones Math. 21 (1973), 213-221. MR 48:9710

[S2] G. Segal, Categories and cohomology theories, Topology 13 (1974), 293-312. MR 50:5782

[V] V. A. Vassiliev, Complements of discriminants of smooth maps: topology and applications, (Translations of Mathematical Monographs, vol. 98), AMS, Providence, 1992. MR 94i:57020

[W] F. Waldhausen, Algebraic K-theory of topological spaces. II, Algebraic Topology. Aarhus 1978. (Lecture Notes in Math; No 763), Springer-Verlag, Berlin a.o., 1979, 356-394. MR 81i:18014b

Institute of Mathematics, Novosibirsk, 630090, Russia

E-mail address: versh@math.nsc.ru 